\title{
LES OPINIONS DES ETUDIANTS SUR LE COURS « EVALUATION ET ADAPTATION DE MATERIELS PEDAGOGIQUES »
}

\section{Cihan AYDOĞU ${ }^{1}$}

\begin{abstract}
Öz: Eğitim fakültelerinin yabancı dil bölümlerinin öncelikli hedefi öğrencileri öğrenim gördükleri yabancı dili etkili bir şekilde öğretmeye hazırlamaktır. Bunun için de, öğrencilerin birçok kuramsal bilginin yanı sıra, bu bilgilerini sınayabileceği bir öğretim ortamına ihtiyacı vardır. Bu bağlamda, eğitim fakültesi bölümlerinin programlarında okul deneyimi ve öğretmenlik uygulaması dersleri yer almaktadır. Ancak sadece birer akademik dönemle sınırlı bu stajlar, öğretmenlik mesleğine hazırlanmada yetersiz kalabilmektedir. Dolayısıyla da öğretmen adaylarına bu gözlem ve uygulamaya dayalı stajlardan önce, yöntem derslerinde bilgilerini sınayabilecekleri bir ortamın yaratılması gerekmektedir. Yaparak öğrenme bu ortamı sağlayabilecek yöntemlerden biri olarak karşımıza çıkmaktadır. Bu çalışmada hedefimiz yaparak öğrenme yöntemini kullanarak bu amaca hizmet etmektir. Bu doğrultuda, Materyal Değerlendirme ve Uyarlama dersinde tek grup son-test modeline dayanarak Fransızca bölümü öğrencilerine özgün dokümanlardan hareketle çalışma yaprakları hazırlattık. Dersler haftada 3 saat olmak üzere toplam 12 hafta sürdü. İlk 4 hafta teorik bilgiler verildi. İzleyen 8 hafta boyunca uygulama çalışmaları gerçekleştirildi. Yaparak öğrenmenin öğrenciler üzerindeki etkilerini ölçmek amaciyla açık uçlu sorulardan oluşan bir anket uygulandı. Sonuç olarak, yaparak öğrenmenin öğrencilerde bir Fransızca yabancı dil dersi hazırlama konusunda birçok beceriyi geliştirdiği ve öğrencilerin özgüvenini desteklediği görülmüştür. Öğrencilerin yaşadıkları güçlükleri tespit etmek maksadıyla sorulan soruya verdikleri cevaplar değerlendirildiğinde ise yaparak öğrenme tekniğinin güçlükleri azaltma yönünde olumlu bir katkısı olduğu ancak yine de bazı güçlüklerin devam ettiği tespit edilmiştir.
\end{abstract}

Anahtar Sözcükler: Çalışma Yaprağı, Yaparak Öğrenme, Yabancı Dil, Materyal Hazırlama, Özgün Doküman, Öğretmen Adayı.

\section{Introduction}

L'objectif primordial des départements de langues des facultés de pédagogie est de former les futurs enseignants de langues étrangères. Pendant ce processus, les étudiants apprennent à planifier une situation d'apprentissage, à organiser des activités d'apprentissage, à faire des évaluations et à contribuer au

\footnotetext{
${ }^{1}$ Yrd. Doç. Dr., Anadolu Üniversitesi, Eğitim Fakültesi, Yabancı Diller Eğitimi Bölümü, Fransız Dili Eğitimi Anabilim Dalı. caydogdu@anadolu.edu.tr
} 
développement sociologique, psychologique, physiologique et cognitif de l'élève. Un bon enseignant ne transmet pas seulement des connaissances. Il doit d'abord être bien motivé pour son métier, aimer ses élèves, créer une bonne relation avec eux, connaître son domaine, avoir des expériences, savoir satisfaire les besoins des élèves, pouvoir résoudre les problèmes vécus en classe, savoir réorganiser son cours d'après les réactions des élèves et avant tout se sentir prêt à enseigner. Donc, les responsabilités d'un enseignant sont d'une importance vitale car l'enseignant est d'une façon l'architecte de l'avenir d'une société et il prépare les élèves au futur.

Un enseignant ne doit pas se contenter d'un manuel imposé par l'établissement scolaire. Avec la technologie qui se développe à grande vitesse, les caractéristiques des élèves changent aussi. Chaque année, on se retrouve avec un groupe différent. Les besoins et les attentes des élèves d'aujourd'hui ne seront pas les mêmes que ceux des élèves d'ici quelques ans, c'est la raison pour laquelle un manuel tout seul ne peut faire le miracle. C'est plutôt la méthode d'apprentissage de l'enseignant qui ouvre la voie de la réussite aux élèves. Déjà, le choix de l' éclectisme se manifeste chez les nouveaux enseignants par un refus délibéré de l'emploi de la méthode ou du manuel tel que l'auteur le préconise ou que l'enseignant standard des générations précédentes le pratique. Contrairement à leurs devanciers, ils n'adoptent plus un manuel mais ils l'adaptent. Ils s'en tiennent rarement à la manipulation d'un seul. Même une tendance qui consiste à estimer que:

Seul un matériel conçu pour un public qui aura défini les besoins et cerné les caractéristiques pourra être efficace, se fait jour. Cette option méthodologique peut aller jusqu'à l'élaboration de matériel didactique au jour le jour à partir de documents et d'activités qui permet à l'enseignant d'être en prise directe sur la réalité d'un groupe d'apprenants (Bérard, 1995, p. 21).

Bien qu'une telle tendance commence à se répandre de plus en plus entre les enseignants, il ne devrait pas être question de rejeter complétement le manuel mais plutôt de le compléter. Car, sans manuel, il pourrait nous arriver de perdre le fil et sans progression il serait bien difficile de décider par où commencer et comment orienter le processus d'enseignement/apprentissage. Déjà les établissements scolaires imposent l'utilisation d'un manuel. Alors, le rôle de l'enseignant est de bien connaître les besoins et les styles d'apprentissage de ses élèves et de compléter les points faibles du manuel qui est conçu pour un public d'élèves général.

Si tel est le cas, un enseignant doit souvent avoir recours à des documents authentiques.

L'emploi des documents authentiques en classe est apparu lorsque les didacticiens se sont préoccupés des apprenants qui étaient au-delà du niveau débutant. Pour ces étudiants qui avaient des aptitudes linguistiques souvent hétérogènes le simple prolongement de la méthodologie employée au Niveau I était inadéquat. Il fallait les faire passer du maniement d'une langue prétendument « neutre » et aux structures langagières limitées à l'acquisition 
d'une compétence linguistique permettant une plus grande liberté d'expression. Faire entrer dans la classe des messages sonores, écrits, visuels ou pluri codés appartenant à l'environnement quotidien des français, était une des manières de tenter de résoudre les problèmes posés par le Niveau II d'apprentissage. Pour les différencier du matériel spécifiquement fabriqué pour la classe, on accola à ces documents non prévus à l'origine pour un usage pédagogique, l'adjectif authentique (Lebre - Peytard, 1990, p. 7).

Pour les partisans des documents authentiques, les avantages de leur utilisation sont l'introduction en classe de fragment d'extrait de l'univers quotidien des Français, la sensibilisation des élèves à des registres de langage diversifiés et aux véritables emplois de la langue française. Ceux qui sont contre leur utilisation prétendent que, une fois introduits dans un cadre pédagogique, ces documents perdent leur label d'authenticité, et qu'il vaut mieux, dans ces conditions, se contenter de documents ad hoc conçu spécialement pour la classe de langue (idem).

Malgré ceux qui contestent, ces dernières années, l'utilité des documents authentiques est devenue incontestable. L'idée unanime est que comprendre un document qui n'a pas été conçu spécialement pour son apprentissage motive les élèves, leur prête l'occasion de s'autoévaluer et à l'enseignant d'évaluer ses élèves, de combler les points faibles d'un manuel et encore permet de créer une interaction entre les élèves eux-mêmes et entre les élèves et l'enseignant.

Par contre, il ne suffit pas d'introduire en classe un bulletin d'informations ou un article de journal pour que la communication s'établisse. Si on se limite dans des exercices de compréhension à de simples questions/réponses et on utilise ces documents pour étudier un point grammatical, il faut s'interroger sur son utilité en classe. A condition de ne pas dévier de sa fonction sociale, comme Lebre-Peytard (1990, p. 9) l'indique « ces documents constituent d'excellents matériaux sociaux qui peuvent améliorer les compétences linguistiques, communicatives et culturelles des apprenants ».

Dans ce cas, pour qu'un document authentique ne perde pas de son authenticité, de sa fonction sociale, il parait indispensable de savoir comment l'utiliser, comment s'en servir à des fins éducatives. A ce point, faire des pratiques avant de commencer à enseigner dans un établissement scolaire devient inévitable. Faire des pratiques constitue l'étape la plus essentielle dans la formation des futurs enseignants. Se contenter seulement des connaissances théoriques ne serait jamais suffisant pour qu'ils puissent connaître efficacement la profession enseignante et provoquerait des difficultés et des échecs pendant son exécution (Oğuz, 2004, p. 143). Cependant faire des pratiques avant de se retrouver avec des élèves permet au futur enseignant de connaître les vraies difficultés, les problèmes qui peuvent surgir et de se préparer à gérer une vraie classe. Et encore, la pratique crée une situation d'apprentissage où les futurs enseignants apprennent à expérimenter les méthodes et techniques d'un apprentissage efficace. 
C'est pour cela que les étudiants en quatrième année de la faculté de pédagogie font des stages dans les écoles. Durant le premier semestre, ils observent les pratiques pédagogiques d'un professeur et au deuxième semestre, ils assistent un professeur. La plus grande utilité des stages est qu'ils prêtent l'occasion de se concentrer non pas sur la question « quoi enseigner ? " mais plutôt sur la question " comment enseigner? », ce qui, avec l'approche communicative et les nouvelles technologies, se situe au cœur de toutes sortes d'activités pédagogiques. Tout au long de ce processus d'expérience, les étudiants apprennent à planifier les activités à faire, la démarche à suivre, les stratégies, méthodes et techniques à utiliser et à penser à l'interaction et l'évaluation (Göçer, 2008 ; Karadüz, Eser, Şahin et İlbay, 2009; Şişman et Acat, 2003).

Il est bien évident que, avant le stage, les étudiants doivent déjà avoir appris à planifier un cours et doivent déjà avoir fait des pratiques, des essais. Donc, les enseignants doivent leur donner l'occasion de faire des pratiques pendant leur formation. Les programmes des départements de langue étrangère des facultés de pédagogie contiennent certains modules contribuant dans ce sens à la formation des futurs enseignants. Le module " évaluation et adaptation de matériels pédagogiques " doit être l'un de ces modules qui leur permet d'acquérir des expériences préprofessionnelles en faisant des pratiques. Car :

L'apprentissage par la pratique est un apprentissage par l'action. Il s'agit d'un processus, pendant lequel les individus construisent des connaissances et reçoivent des acquis et des valeurs d'une expérience directe. L'apprentissage par l'expérience a lieu dès lors que les individus sont impliqués dans une certaine activité, reflètent de manière critique cette activité, retirent de son analyse des perceptions utiles, et utilisent les résultats pour changer une compréhension et/ou un comportement (Académie Internationale d'Apprentissage par la Pratique, 2012, p. 1).

Il est vrai qu'il faut pratiquer et répéter pour apprendre à savoir faire mais on peut pratiquer et répéter les mêmes erreurs. La pratique toute seule n'apporte pas toujours le bon résultat.

L'apprentissage par la pratique doit aussi inclure l'analyse de l'action. C'est l'analyse de ce que l'on fait dans l'action qui permet la correction et la généralisation à plusieurs autres situations. C'est au moment de l'analyse que les étudiants revoient leur procédure, adaptée ou non, et utilisent les concepts nécessaires à la bonne compréhension de la situation et de la procédure. Cependant, agir et analyser ne suffisent pas encore pour un apprentissage maximal.

Pour bien apprendre par la pratique, l'action et l'analyse doivent être précédées de l'anticipation. Pendant cette anticipation, l'étudiant utilise ses préconceptions et ses intuitions. En demandant à l'étudiant de justifier ses anticipations, il fait appel (ou non) aux connaissances déclaratives déjà vues (Gagnon, 2012, p. 1).

Bref, l'apprentissage par la pratique n'est pas une technique qui ne consiste qu'à faire des pratiques et des répétitions. C'est un apprentissage qui a lieu à la suite 
de l'utilisation d'informations accompagnée d'une réflexion sur l'effet produit par cette action en fonction des buts visés.

\section{L'objectif de la recherche}

L'objectif principal de cette recherche est de déterminer les apports de la théorie d'apprentissage par la pratique au cours de «Evaluation et Adaptation de Matériels Pédagogiques » aux futurs professeurs de français langue étrangère.

Dans le cadre de cet objectif, nous chercherons à répondre aux questions suivantes :

1. Quelles sont les opinions des étudiants sur les effets et les apports de l'Apprentissage par la Pratique?

1.1. Est-ce que les étudiants croient à l'utilité de l'Apprentissage par la Pratique?

1.2. Est-ce que l'Apprentissage par la Pratique contribue à la confiance en soi des étudiants?

1.3. Est-ce que les étudiants apprécient vraiment les feed-back donnés pendant leurs présentations?

\section{Méthode de la recherche}

\subsection{Le modèle de la recherche}

Dans cette recherche pour voir l'apport et les effets de l'utilisation de l'apprentissage par la pratique au cours d'évaluation et d'adaptation de matériels pédagogiques nous avons appliqué le modèle de recherche " groupe unique avec post-test ».

Le modèle de recherche " groupe unique avec post-test » est la méthode expérimentale la plus convenable quand les chercheurs travaillent avec un seul et petit groupe dans une certaine durée et avec un seul post-test ou observation (Laveault, 1997 ; Ergun, 2012).

Dans cette recherche, nous avons eu recours à la méthode qualitative pour connaitre les opinions des étudiants concernant le cours d'évaluation et d'adaptation de matériels pédagogiques. Pour cette raison, à la fin de l'expérimentation, nous avons demandé aux étudiants du groupe expérimental de répondre à un questionnaire composé de questions ouvertes.

Les étudiants qui ont participé à cette recherche empirique basée sur l'utilisation du modèle de recherche " groupe unique avec post-test " étaient tous des volontaires.

\subsection{L'univers de la recherche}

Les étudiants de la quatrième année du département de Français de la faculté de pédagogie de l'université Anadolu constituent l'univers de cette recherche. 18 étudiants ont suivi le cours " évaluation et adaptation de matériels pédagogiques».

Jusqu'à la quatrième année les étudiants ont déjà suivi des cours théoriques et pratiques sur l'enseignement des langues étrangères. Nous estimons qu'ils 
connaissent les méthodologies, les caractéristiques des quatre habiletés, les types d'activités, les caractéristiques propres aux enfants et ont déjà des connaissances sur comment diriger une classe, guider et évaluer les élèves et préparer des matériels visuels, sonores et écrits.

\subsection{La collection des données}

Pour collecter les données de cette recherche, nous avons utilisé un questionnaire. Le questionnaire est préparé d'après les objectifs visés par un apprentissage par la pratique. Les recherches faites dans ce cadre prétendaient que cette méthode d'apprentissage augmenterait la confiance en soi des étudiants, que les étudiants recevraient des acquis par une expérience directe, qu'ils trouveraient l'occasion de pratiquer leurs connaissances théoriques, qu'avec le temps passé les difficultés diminueraient et qu'un apprentissage pratique devrait inclure l'analyse de l'action réalisée pour éviter la répétition des mêmes erreurs. Par conséquent, pour pouvoir tester ces apports attendus d'un apprentissage par la pratique, nous avons formulé 4 questions ouvertes et nous avons eu recours au point de vue de trois experts en ce domaine. La première question a pour but de découvrir les opinions des étudiants à propos du cours " évaluation et adaptation de matériels pédagogiques ». La deuxième question a été préparée pour observer la confiance en soi des étudiants après avoir travaillé avec la technique d'apprentissage par la pratique dans ce cours. La troisième question vise à définir les effets des feed-back donnés par l'enseignant et les autres étudiants pendant la présentation des fiches pédagogiques. La dernière question a pour objectif de préciser les difficultés vécues par les étudiants.

Notre questionnaire se compose des 4 questions suivantes préparées afin de trouver des réponses aux questions concernant les objectifs de la recherche.

1- Quels sont les apports de l'utilisation de la technique d'Apprentissage par la Pratique dans le cours « Evaluation et Adaptation de Matériels Pédagogiques »? Qu'est-ce que vous avez appris à faire pendant ce cours ?

2- Si vous comparez les sentiments que vous avez vécus pendant la préparation ou la présentation de votre fiche au début et à la fin du semestre, quels changements vous observez chez vous-même?

3- Comment pourrez-vous définir les effets et les apports des feed-back donnés par l'enseignant et par vos camarades de classe ? Comment vous vous en êtes servi ?

4- Avez-vous vécu des difficultés pendant la présentation ou la préparation de vos matériels pédagogiques? Si oui, expliquez clairement vos difficultés.

Comme les étudiants étaient en quatrième année, nous avons posé les questions directement en français. Nous ne nous sommes pas inquiétés de leur compréhension. Mais pour les réponses, nous les avons laissés libres en disant qu'ils pourraient répondre soit en turc soit en français pour qu'ils puissent s'exprimer clairement et correctement. Après avoir recueilli le questionnaire de 
chaque étudiant, nous avons remarqué qu'ils avaient tous préféré répondre en leur langue maternelle, en turc.

\subsection{L'expérience}

L'expérimentation s'est déroulée pendant le premier semestre de l'année universitaire 2012-2013 à raison de 3 heures de cours par semaine dans le cadre du cours «Evaluation et adaptation de matériels pédagogiques » pendant 12 semaines avec les étudiants de FLE en quatrième année de licence. Durant cette recherche nous avons travaillé avec un seul groupe appelé " groupe expérimental », composé de 18 étudiants.

La première semaine nous avons expliqué le déroulement du cours et fait une courte révision sur les méthodologies d'enseignement des langues étrangères. La deuxième semaine nous avons discuté le rôle, l'importance et la diversité des documents authentiques. La troisième semaine nous avons discuté les objectifs à viser dans un cours de langue d'après les critères du Cadre Européen Commun de Référence pour les Langues et l'importance de l'âge et du centre d'intérêt des élèves lors du choix d'un document authentique. La quatrième semaine nous avons analysé des exemples de fiches pédagogiques en nous basant aussi sur la question « comment formuler une consigne et définir un objectif ?».

Dans ce cours, au lieu de faire travailler les étudiants sur différents types d'activités séparément, nous avons privilégié le travail autour d'un document précis et sous forme d'une fiche pédagogique. Car nous avons pensé que préparer la fiche pédagogique d'un document (photo, chanson...) permettrait aux étudiants de faire des pratiques à planifier un cours, définir les objectifs, préparer des activités, décider de la durée et penser à l'évaluation formative.

En raison d'une durée limitée et pour donner à chaque étudiant l'occasion de travailler sur plusieurs documents, il nous a paru plus convenable de les faire travailler en groupe. Pour la formation des groupes, nous avons demandé aux étudiants de former 6 sous-groupes de 3. A ce stade nous les avons laissés libres. Car, il fallait qu'ils se retrouvent en dehors de la classe pour préparer leur matériel. Alors, pour qu'ils puissent s'organiser facilement, on leur a donné l'occasion de se grouper par affinités de personnes. Dans ces groupes il n'y avait pas de leader, tous les étudiants étaient sur un pied d'égalité.

Pendant les 8 autres semaines, les étudiants ont fait la présentation de leur fiche pédagogique. Pour se préparer et pour qu'ils aient une idée précise du travail demandé, nous leur avons offert des fiches exemplaires et une liste d'activités possibles pour chacun des documents à préparer. Avant de commencer à présenter leur travail, nous avons posé aux étudiants les questions suivantes ;

-Quels sont les objectifs à atteindre ?

-Avec les élèves de quel âge supposez-vous travailler ?

-Quel est l'intérêt de ce document?

-Quelles sont les connaissances antérieures nécessitées par ce document? 
Chaque semaine les 3 groupes ont présenté les fiches qu'ils avaient préparées auparavant. La semaine suivante les 3 autres groupes ont fait leur présentation préparée à partir du même type de matériel. Comme les présentations duraient 2 semaines, pendant 8 semaines nous avons pu travailler sur 4 types de document ; photo/image, brochure, chanson, bande dessinée. Pendant la présentation d'un groupe, nous et les autres étudiants avons donné des feed-back sur la planification, les types d'activités choisis, les objectifs visés et les points auxquels ils devraient faire attention pour la prochaine fois. Pendant cette phase nous avons posé des questions aux étudiants concernant leur fiche et leur avons donné l'occasion de se justifier.

En dehors des travaux de groupe, les étudiants ont aussi préparé des fiches individuelles. Car il fallait qu'ils soient notés. Pour les deux examens partiels et pour l'examen final ils ont préparé des fiches individuellement. Donc au total, ils ont préparé sept fiches pédagogiques dont quatre en groupe et trois individuellement.

A la fin du semestre, les membres des groupes ont répondu individuellement chez eux à un questionnaire composé de 4 questions ouvertes. On ne leur a pas demandé de le faire en classe car nous voulions qu'ils se sentent à l'aise et qu'ils ne soient pas influencés l'un par l'autre. L'analyse des résultats a été faite à partir des réponses des 18 étudiants du groupe de travail.

\section{Présentation et analyse des résultats}

Dans cette partie, nous allons analyser les données recueillies de l'expérimentation menée dans le cours de FRÖ 405 Evaluation et Adaptation de Matériels Pédagogiques lors de la session d'automne de l'année universitaire 2012-2013 dans le département de Français Langue Etrangère de l'université Anadolu.

\subsection{Le mode d'analyse des données}

Pour analyser les données obtenues du questionnaire, en raison du nombre limité d'étudiants (18 étudiants), nous avons eu recours à la fréquence. Nous avons compté les fréquences d'apparition des énoncés les plus prononcées par les étudiants et nous les avons présentées sous forme de tableau.

\subsection{Les résultats obtenus du questionnaire}

L'analyse de ce questionnaire nous montre les effets de la technique d'apprentissage par la pratique dans le cours d' «évaluation et adaptation de matériels pédagogiques » et les opinions des apprenants à propos de ce cours enseigné avec la technique d'apprentissage par la pratique.

Nous avons procédé à notre analyse à partir de 3 catégories thématiques.

A. Les apports de la technique d'apprentissage par la pratique concernant le cours « évaluation et adaptation de matériels pédagogiques ».

B. Les apports des feed-back donnés par l'enseignant et par les autres étudiants de la classe. 
C. Les difficultés rencontrées lors de l'utilisation de la technique d'apprentissage par la pratique.

Selon les réponses données par les étudiants, pour chaque catégorie thématique, nous avons formé des sous-thèmes. Les fréquences d'apparition et les apports les plus prononcés par les étudiants sont présentés dans les tableaux qui suivent.

Tableau 1: Distribution des sous-thèmes de la catégorie thématique A (Les apports de la technique d'apprentissage par la pratique concernant le cours "évaluation et adaptation de matériels pédagogiques »)

\begin{tabular}{|l|c|}
\hline $\begin{array}{l}\text { A1- Les apports concernant le cours « évaluation et adaptation de } \\
\text { matériels pédagogiques » }\end{array}$ & N/ 18 \\
\hline $\begin{array}{l}\text { A1.1- L'apprentissage par la pratique aide à apprendre à planifier un cours de } \\
\text { français langue étrangère }\end{array}$ & 18 \\
\hline A1.2- L'apprentissage par la pratique aide à préciser la durée d'une activité & 13 \\
\hline $\begin{array}{l}\text { A1.3- L'apprentissage par la pratique permet à l'étudiant de pouvoir fixer les } \\
\text { objectifs communicatifs et linguistiques }\end{array}$ & 16 \\
\hline $\begin{array}{l}\text { A1.4- L'apprentissage par la pratique développe chez l'étudiant l'aptitude de } \\
\text { choisir le matériel le plus convenable au niveau des apprenants }\end{array}$ & 15 \\
\hline $\begin{array}{l}\text { A1.5- L'apprentissage par la pratique sensibilise l'étudiant à suivre les trois } \\
\text { étapes d'une fiche pédagogique: pré-activité, pendant activité, post activité }\end{array}$ & 18 \\
\hline $\begin{array}{l}\text { A1.6- L'apprentissage par la pratique rend l'étudiant capable d'organiser les } \\
\text { activités du général au spécifique et du simple au complexe }\end{array}$ & 18 \\
\hline $\begin{array}{l}\text { A1.7- L'apprentissage par la pratique aide à apprendre à formuler les } \\
\text { consignes d'une façon claire et précise }\end{array}$ & 14 \\
\hline
\end{tabular}

Comme le montre le tableau 1 , d'après les réponses données au questionnaire, nous pouvons dire qu'avec l'apprentissage par la pratique les étudiants apprennent à planifier un cours de français langue étrangère. D'ailleurs tous ont précisé qu'ils ont appris à planifier un cours dans leur réponse à la première question du questionnaire (Quels sont les apports de l'utilisation de la technique d'apprentissage par la pratique dans le cours d'évaluation et adaptation de matériels pédagogiques? Qu'est-ce que vous avez appris à faire dans ce cours ?).

D'autre part, d'après les réponses à la première question 13 étudiants pensent qu'après ce cours ils arriveront plus facilement à préciser la durée d'une activité. Les 5 autres n'ont pas donné leurs opinions sur ce sujet. D'après nos observations pendant le cours, au début, les étudiants éprouvaient trop de difficultés à définir les objectifs. Mais comme il est indiqué dans le tableau 1, la plupart des étudiants (16 étudiants) disent que dans ce cours ils ont appris à définir les objectifs communicatifs et linguistiques.

Toujours selon le tableau 1, nous pouvons prétendre que la plupart des étudiants (15 étudiants) ont appris à choisir un document authentique à partir du public visé, que tous les étudiants sont devenus bien conscients des trois étapes (préactivité, activité, post-activité) d'une fiche pédagogique et qu'ils ont compris l'importance d'aller du général au spécifique et du simple au complexe. Le 
dernier apport cité par les étudiants (14 étudiants) est qu'ils ont appris à formuler les consignes d'une façon claire et précise.

Tableau 2: Distribution des sous-thèmes de la catégorie thématique A (Les apports de la technique d'apprentissage par la pratique concernant le cours "évaluation et adaptation de matériels pédagogiques »)

\begin{tabular}{|l|c|}
\hline A2- Les apports concernant la confiance en soi des apprenants & N/ 18 \\
\hline $\begin{array}{l}\text { A2.1- L'apprentissage par la pratique donne aux étudiants l'occasion } \\
\text { d'apprendre à contrôler leurs sentiments }\end{array}$ & 12 \\
\hline $\begin{array}{l}\text { A2.2- L'apprentissage par la pratique permet aux étudiants d'auto évaluer } \\
\text { leurs progrès }\end{array}$ & 16 \\
\hline $\begin{array}{l}\text { A2.3- L'apprentissage par la pratique apprend aux étudiants de s'exprimer } \\
\text { plus aisément devant un groupe }\end{array}$ & 18 \\
\hline $\begin{array}{l}\text { A2.4- L'apprentissage par la pratique contribue à l'estime de soi des } \\
\text { étudiants }\end{array}$ & 17 \\
\hline $\begin{array}{l}\text { A2.5- L'apprentissage par la pratique aide les étudiants à se préparer à } \\
\text { être professeur de français langue étrangère }\end{array}$ & 15 \\
\hline $\begin{array}{l}\text { A2.6- L'apprentissage par la pratique aide les étudiants à se sentir plus } \\
\text { prêt à compléter les points faibles d'un manuel }\end{array}$ & 18 \\
\hline
\end{tabular}

Le tableau 2 présente les réponses que les étudiants ont données à la deuxième question du questionnaire ( $\mathrm{Si}$ vous comparez les sentiments que vous avez vécus pendant la préparation ou la présentation de votre fiche au début et à la fin du semestre, quels changements vous observez chez vous-même ?). 12 étudiants disent qu'ils étaient très émus et stressés pendant leur présentation au début du semestre mais qu'avec le temps ils ont appris à contrôler leurs sentiments. Encore d'après les réponses on peut dire que l'apprentissage par la pratique donne aux étudiants (16 étudiants) l'occasion d'évaluer leurs propres progrès, ce qui est une étape essentielle et nécessaire dans l'enseignement/apprentissage, et que, autant qu'ils vivent des expériences les étudiants (18 étudiants) arrivent à s'exprimer plus aisément devant un groupe.

Toujours selon le deuxième tableau, au fur et à mesure que les étudiants préparent des fiches pédagogiques, ils (15 étudiants) se sentent plus prêts à enseigner le français en tant que langue étrangère et à combler (18 étudiants) les points faibles d'un manuel en préparant des fiches pédagogiques à partir des documents authentiques actuels.

Tableau 3: Distribution des sous-thèmes de la catégorie thématique $B$ (Les apports des feed-back donnés par l'enseignant et par les autres apprenants de la classe)

\begin{tabular}{|l|c|}
\hline B- Les apports concernant les feed-back & N/ 18 \\
\hline B1- Les feed-back permettent aux étudiants de se perfectionner & 18 \\
\hline B2- Les feed-back créent une progression pas à pas & 15 \\
\hline B3- Les feed-back assurent la diminution des fautes & 16 \\
\hline B4- Les feed-back élimine la répétition des mêmes erreurs & 16 \\
\hline B5- Les feed-back permettent l'apprentissage de l'auto correction & 18 \\
\hline
\end{tabular}




\begin{tabular}{|l|c|}
\hline B6- Les feed-back permettent de trouver des activités plus créatives & 11 \\
\hline $\begin{array}{l}\text { B7- Les feed-back rendent les étudiants plus conscients du travail à } \\
\text { effectuer }\end{array}$ & 18 \\
\hline
\end{tabular}

Le tableau 3 présente les apports des feed-back. Les données sont obtenues à partir des réponses à la question «Comment pouvez-vous définir les effets et les apports des feed-back donnés par l'enseignant et vos camarades de classe? Comment vous vous en êtes servi ? ». 18 étudiants disent qu'à partir des feedback donnés, soit par l'enseignant soit par les autres étudiants, ils apprennent à quoi ils devraient faire plus attention, ils se rendent compte de leurs points faibles et ils apprennent à résoudre leurs problèmes. D'où on peut prétendre que les feed-back permettent aux étudiants de se perfectionner.

D'autre part, 15 étudiants ont écrit qu'ils apprenaient à fixer des objectifs, à planifier un cours de français langue étrangère, qu'ils se rendaient compte des critères à prendre en considération pour le choix d'un document authentique et qu'ils veillaient à donner lieu à différents types d'activité. Et encore, ils ont ajouté qu'ils arrivaient à résoudre pas à pas les problèmes qu'ils éprouvaient juste au début du semestre et qu'ils arrivaient plus facilement à préparer une fiche pédagogique. Cela nous permet de parler d'une progression "pas à pas » grâce à l'apprentissage par la pratique.

Toujours concernant les apports des feed-back, 16 étudiants précisent que grâce aux feed-back ils ont fait chaque semaine moins de fautes, que leur fiche était moins critiquée, qu'ils (16 étudiants) ne répétaient pas les mêmes erreurs et aussi, qu'ils (18 étudiants) les remarquaient eux-mêmes et qu'ils arrivaient à les corriger tout seul.

Seulement 11 étudiants ont écrit qu'au début ils mettaient plutôt des activités structurelles dans leur fiche mais qu'avec le temps ils ont appris à préparer des activités plus créatives.

Dernièrement nous pouvons dire que donner à l'étudiant l'occasion de mettre en pratique ses connaissances théoriques lui permet de concrétiser ses savoirs. Car les 18 étudiants disent que grâce à des pratiques, ils sont devenus plus conscients du travail à faire et des exigences du métier de professeur de français langue étrangère.

Tableau 4: Distribution des sous-thèmes de la catégorie thématique C (Les difficultés rencontrées lors de l'utilisation de la technique d'apprentissage par la pratique)

\begin{tabular}{|l|c|}
\hline $\begin{array}{l}\text { C- Les difficultés rencontrées dans le cours de « évaluation et } \\
\text { adaptation de matériels pédagogiques }\end{array}$ & N/ 18 \\
\hline $\begin{array}{l}\text { C1- Les étudiants éprouvent des difficultés à trouver le matériel } \\
\text { convenable aux objectifs d'enseignement }\end{array}$ & 13 \\
\hline C2- Les étudiants ont des difficultés à diversifier les types d'activités & 8 \\
\hline $\begin{array}{l}\text { C3- Les étudiants ont des difficultés à se réunir avec les autres membres } \\
\text { du groupe }\end{array}$ & 5 \\
\hline C4- Les étudiants vivent des difficultés à trouver un matériel convenable & 7 \\
\hline
\end{tabular}




\begin{tabular}{|l|c|}
\hline au public; âge, centre d'intérêt, expérience etc... & 5 \\
\hline $\begin{array}{l}\text { C5- Les étudiants ont des difficultés à se mettre d'accord avec les autres } \\
\text { membres du groupe }\end{array}$ & 6 \\
\hline $\begin{array}{l}\text { C6- Les étudiants éprouvent des difficultés à écrire des consignes claires } \\
\text { et précises }\end{array}$ & 6 \\
\hline
\end{tabular}

Dans cette recherche nous avons aussi voulu observer si l'application de l'apprentissage par la pratique pose des difficultés pour l'étudiant. Le tableau 4 présente les réponses que les étudiants ont données à la question «Avez-vous vécu des difficultés avant et pendant la présentation ou la préparation de vos matériels pédagogiques ? Si oui, expliquez clairement vos difficultés ».

A partir des réponses des étudiants, il est possible de regrouper les difficultés en six sous-catégories. La plus grande difficulté qu'ils (13 étudiants) éprouvent est de trouver le matériel convenable aux objectifs à enseigner. 8 étudiants ont écrit qu'ils avaient souvent tendance à faire les mêmes types d'activité, ce qui crée une monotonie dans la classe, et qu'ils avaient du mal à créer des activités de différents types.

Une autre difficulté prononcée par les 5 étudiants est qu'ils ont vécu des difficultés à se retrouver avec leurs partenaires pour préparer leur fiche pédagogique. Encore 7 étudiants ont écrit qu'il leur était très difficile de trouver un document authentique qui correspondait bien à l'âge, au centre d'intérêt ou à l'expérience vécue du public visé et encore de décider parmi les choix.

Une autre difficulté remarquée dans les réponses des étudiants concerne l'accord entre les membres du groupe. 5 étudiants ont écrit qu'ils ont eu du mal à se mettre d'accord sur le matériel et les activités à faire.

La dernière difficulté éprouvée par les étudiants concerne les consignes des activités. 6 étudiants disent que malgré plusieurs pratiques, ils ont toujours du mal à formuler les consignes d'une façon claire et précise.

\section{Conclusion/Discussion}

Les résultats de cette étude nous permettent de prétendre que l'apprentissage par la pratique rend les futurs enseignants de langue capable à planifier un cours, à fixer les objectifs, à préparer des activités, à organiser les activités entre elles allant du simple au complexe et du général au spécifique, à formuler des consignes et à penser à l'évaluation formative. De plus, il contribue au développement de leur confiance en soi et leur permet de s'autoévaluer.

Ceci s'expliquerait par la contribution des feed-back qui ont permis aux étudiants de connaître leurs points faibles et leurs points forts, ce qui les a aidés à se perfectionner suivant une progression «pas à pas ». Voir la diminution des erreurs commises par rapport à la fiche pédagogique précédente les a bien motivés et les a incités à viser le meilleur.

Dans ce travail réalisé avec les futurs enseignants de FLE, les étudiants ont trouvé l'occasion de s'habituer à s'exprimer devant un groupe, à contrôler leurs sentiments et de se faire des expériences avant de faire leur stage dans les 
écoles. Ils ont au moins obtenu l'occasion de vivre une situation de classe et ont connu les exigences du métier d'enseignant de langue, et sont devenus conscients de ce qu'ils devaient faire pendant leur stage et plus tard, quand ils exerceront le métier.

Quant aux difficultés rencontrées, les résultats obtenus nous permettent de prétendre que les étudiants n'ont pas vécu de grandes difficultés pendant le processus. On constate que la difficulté le plus souvent citée par les étudiants consiste à trouver le matériel le plus approprié aux objectifs d'enseignement. Mais même les enseignants qui ont plusieurs années d'expérience peuvent, de temps en temps, rencontrer des difficultés pareilles quand ils veulent travailler avec des documents authentiques, car il n'est pas toujours si facile de trouver un document authentique adéquat au niveau de connaissance, aux besoins des étudiants et aux objectifs du cours.

En somme, nous pouvons dire que l'apprentissage par la pratique incite les étudiants à devenir actif, ce qui entraine la motivation et les empêche de rester spectateur de leur apprentissage. Car c'est eux qui décident du contenu et du déroulement du cours ainsi que du matériel à utiliser. De plus, ils s'auto évaluent et se corrigent eux-mêmes avec la collaboration de l'enseignant et des autres étudiants. L'apprentissage par la pratique permet donc aux étudiants de mettre la main à la pâte et d'expérimenter leurs connaissances théoriques.

Les résultats obtenus de ce travail, nous permettent de prétendre qu'il faut donner aux étudiants des départements de langue plus d'occasion pour pratiquer et tester leurs connaissances sur l'enseignement/apprentissage d'une langue étrangère avant d'aller faire leur stage aux écoles. Pendant ce travail, nous n'avons pu travailler qu'avec quatre types de documents authentiques et au long d'un seul semestre. Mais nous supposons que travailler d'une plus longue durée et sur plusieurs autres types de documents contribueraient à la diminution des difficultés annoncées par les étudiants et à leur confiance en soi.

\section{BIBLIOGRAPHIE}

Académie Internationale d'Apprentissage par la Pratique. (2008). " Qu'est-ce que l'Apprentissage et l'Education par la Pratique ? ". Date d'accès: 10 Octobre 2012, http://www.viaexperientia.net/uploads/1.via_borchure_draftLT.pdf.

Annie, F. (2011). Effets des Pratiques Pédagogiques sur les Apprentissages. Institut Français de l'Education: Agence Qualité Education, 65.

Bérard, E. (1995). L'approche Communicative. Paris: CLE International.

Courtillon, J. (2003). Elaborer un Cours de FLE. Paris: Hachette.

Cuq, J.-P. et Gruca, I. (2003). Cours de Didactique du Français Langue Etrangère et Seconde. France: PUG.

Ergun, M. (2012). "Bilimsel Araştırma Yöntemleri”. Erişim Tarihi: 12 Aralık 2012, www.eğitim.akü.edu.tr/arastırmadesenleri1.ppt. 
Gagnon, C. (2012). “Comment Apprendre par la Pratique?”. Date d'accès: 10 Octobre 2012 , rdl.qc.ca/pdf/etudiantplus/commentapprendrepratique.pdf. http://sdp.cegep-

Göçer, A. (2008). "Eğitim Fakültesi Öğrencilerinin Türkçe Öğretimi Bakımından Öğretmenlik Mesleğine Hazır Bulunuşluk Düzeyleri (Niğde Üniversitesi Örneği)" [Elektronik Sürüm]. Çă̆daş Ĕgitim Dergisi, 33 (358), 513.

Karadüz, A., Eser, Y., Şahin, C. ve İlbay, A. B. (2009). "Eğitim Fakültesi Son Sınıf Öğrencilerinin Görüşlerine Göre Öğretmenlik Uygulaması Dersinin Etkililik Düzeyi" [Elektronik Sürüm]. Mustafa Kemal Üniversitesi Sosyal Bilimler Enstitüsü Dergisi, 6 (11), 442-455.

Laveault, D. (1997). "Introduction à la Recherche". Date d'accès: 12 Décembre 2012, http://www.courseweb.uottawa.ca/EDU5590/Pdf/wbloc6h.PDF.

Lebre-Peytard, M. (1990). Situations d'Oral. Documents Authentiques : Analyse et Utilisation. Paris: CLE International.

Ministère d'Education Nationale. (2013). "Les Pratiques d'Enseignement et d'Apprentissage en Dispositif Relais". Date d'accès: 15 Mai 2014, http://cache.media.education.gouv.fr/file/202/52/4/DEPP-dossier-2013-202pratiques-dispositif-relais_246524.pdf.

Ministère de 1'Education. (2000). "Éducation Coopérative et autres Formes d'Apprentissage par l'Expérience". Date d'accès: 10 Janvier 2013, http://www.edu.gov.on.ca/fre/document/curricul/secondary/coop/coopedf.pdf.

Oğuz, A. (2004). "Okul Deneyimi I Dersinin Öğretmen Adayları Üzerindeki Etkileri” [Elektronik Sürüm]. Dumlupınar Üniversitesi Sosyal Bilimler Dergisi, 11, 141-162.

Porcher, L. (2004). L'Enseignement des Langues Etrangères. Paris: Hachette Education.

Reese, H. W. (2011). "The Learning-by-Doing Principle" [Electronic Version]. Behavioral Development Bulletin, 11, 1-19.

Şişman, M. ve Acat, B., M. (2003). "Öğretmenlik Uygulaması Çalışmalarının Öğretmenlik Mesleğinin Algılanmasındaki Etkileri”. [Elektronik Sürüm]. Fırat Üniversitesi Sosyal Bilimler Dergisi, 13 (1), 235-250.

Walters, D. (2005). “John Dewey's Theories of Education”. Date of Access: 25 May 2014, George Novak Internet Archive:https://www.marxists.org/archive/novack/Works/1960/x03.htm.

\section{STUDENT PERCEPTIONS ON THE MATERIALS DEVELOPMENT AND EVALUATION COURSE}

Abstract: The primary objective of the foreign language departments of education faculties is to prepare their students to teach the foreign language effectively. The students not only need to have theoretical courses but also some courses that they can put into practice. To provide 
that need education faculties have school observation and teaching practice courses in their educational programs. Those courses are limited to only one semester and may not be very sufficient to prepare those students to their future professions. Before actual teaching and practicum, students need to have some application courses to apply their methodological knowledge. Learning by doing is one of the methods students can apply their theoretical knowledge into practice. In this study, learning by doing method was used to achieve this goal. This is a single group post-test study design in which students in the French department were asked to prepare worksheets using authentic materials in the Material Evaluation and Adaptation course. It took 12 weeks, 3 hourperiod for each week. The theoretical part took 4 weeks, and the practical activities were carried out during the following 8 weeks. In order to measure the effects of learning by doing method, students were administered a questionnaire consisting of open-ended questions. The results of the study revealed improvements in students' skills in preparing a French language lesson as well as developments in their selfconfidence. The students were questioned to find out the difficulties that they had experienced. The result of this questioning reveals that learning by doing eliminated lots of difficulties to a great extent but still some of the difficulties remained as unsolved.

Keywords: Worksheets, Learning by Doing, Foreign Language, Preparing Materials, Authentic Document, Student Teacher. 